\section{Laurent Degos Sylvie Castaigne Pierre Fenaux Marie-Thérèse Daniel Christine Chomienne}

\title{
Le traitement des leucémies aiguës à promyélocytes par l'acide tout-trans rétinoïque
}

La leucémie aiguë promyléocytaire est une leucémie rare, grave, une urgence thérapeutique en raison du syndrome de coagulation intravasculaire disséminée (CIVD). Cette leucémie est associée à une anomalie cytogénétique, la translocation $\mathrm{t}(15 ; 17)$. L'acide tout-trans rétinoïque est capable, dans la majorité des cas, au moment du diagnostic ou lors de la première rechute, d'induire une rémission complète par différenciation de la cellule maligne. Ces résultats sont obtenus sans phase d'aplasie médullaire, par un traitement per os à domicile. Malheureusement, des rechutes surviennent au bout de quelques mois, nécessitant d'instituer un traitement complémentaire incluant la chimiothérapie cytolytique. L'effet de l'acide rétinoïque dans cette forme de leucémie constitue le premier modèle de traitement par différenciation de la cellule maligne. Cet effet est très probablement dû à l'anomalie moléculaire associée à la translocation chromosomique : un remaniement du gène codant pour le récepteur $\alpha$ de l'acide rétinoïque et aboutissant à la synthèse d'un récepteur hybride. Les mécanismes précis de la cancérisation et de l'action thérapeutique de l'acide tout-trans rétinoïque restent inconnus.

\footnotetext{
ADRESSES

L. Degos : professeur des universités, praticien hospitalier. S. Castaigne : praticien hospitalier. Département d'hćmatologic clinique, hôpital Saint-Louis, 1, avenue Claude-Vellefaux, 75010 Paris, France. P. Fenaux : praticien hospitalier. Service d'hématologie, hôpital Claude-Huriez, 59037 Lille Cedex, France. M.T. Daniel, maître de conférence des universilés, praticien hospilalier. Laboratoire central d'hćmatologie, hôpital Saint-Louis, 1, avenue Claude-Vellefaux, 75010 Paris, France. C. Chomienne : maitre de conférence des uni versité, praticien hospitalier. Inserm U.204, hôpital Saint-Louis, 1, avenue Claude Vel-
}

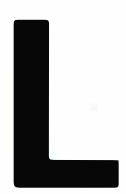

a malignité est le résultat d'un déséquilibre entre prolifération et différenciation cellulaire. Les cellules malignes sont généralement éliminées par chirurgie ou par des agents cytotoxiques, comme la chimiothérapie, la radiothérapie ou l'immunothérapie. Une autre voie de traitement pourrait être une action directe sur la cellule maligne afin d'induire un changement dans le programme cellulaire. Des études in vitro ont démontré la capacité de lignées de cellules malignes à changer leur phénotype soit par inhibition de la prolifération, soit par engagement vers la différenciation [1]. Cette capacité à se différencier est beaucoup plus difficile à obtenir sur des cellules fraîches malignes.

La première orientation choisie pour tenter d'induire in vivo une différenciation des cellules malignes a été de freiner leur prolifération. Le médicament principalement utilisé a été la 
cytosine arabinoside à faible dose [2]. Une étude récente multicentrique chez les personnes âgées atteintes de leucémie aiguë myéloblastique a confirmé l'efficacité de ce traitement [3]. Il est cependant difficile de faire la part entre l'inhibition de prolifération et la cytotoxicité lorsque des agents antimitotiques sont utilisés.

La deuxième orientation vise à changer le programme cellulaire afin de rompre une boucle autocrine de croissance. Un exemple de ce traitement est donné par l'effet de l'interféron $\alpha$ sur les tricholeucocytes. Dans le cas de leucémie à tricholeucocytes, la cellule maligne possède un récepteur du facteur de croissance BCGF ( $B$ cell growth factor) alors que la cellule elle-même émet ce facteur. L'interféron $\alpha$ inhibe l'effet du BCGF [4] et la cellule maligne devient ainsi insensible à ce facteur. Dans cet exemple, la prolifération n'est pas inhibée par un agent extérieur mais par un changement du programme d'expression génique.

$\mathrm{La}$ troisième orientation cherche enfin à induire une différenciation terminale aboutissant à la mort cellulaire. C'est ainsi que les dérivés de l'acide rétinoïque agissent sur la cellule de la leucémie aiguë à promyélocytes, le traitement conduisant à la maturation des cellules malignes. L'acide tout-trans rétinoïque semble constituer, dans les leucémies aiguës à promyélocytes, le premier exemple d'un traitement différenciateur donnant un pourcentage élevé de rémissions complètes.

\section{Les rétinoïdes}

Les rétinoïdes sont des dérivés de la vitamine $\mathrm{A}$, alcool gras soluble. In vivo, cette vitamine (rétinol) est métabolisée de façon réversible en aldéhyde (rétinal) qui peut ensuite être oxydé d'une manière irréversible en acide rétinoïque.

La vitamine A subit des modifications dans l'intestin, puis est transportée par les chylomicrons jusqu'aux vaisseaux lymphatiques et, enfin, par le système porte, jusqu'au foie où le rétinol est stocké sous forme de rétinyl-ester. Il est relargué par le foie, en association avec une protéine porteuse (retinol-binding-protein ou RBP) qui circule avec la pré$\mathrm{m} / \mathrm{s} n^{\circ} .5$, vol. 7. mai 91 albumine (ou transtyrétine). La cellule extrait le rétinol de la circulation tout en relâchant la RBP. Les acides rétinoïques, dont la concentration plasmatique est très faible comparée à celle des rétinols, sont transportés en association très forte avec l'albumine $(99,9 \%)$. Dans la cellule, le rétinol est lié à une protéine porteuse appelée cellular retinol binding protein ou CRBP. Les acides rétinoïques pénètrent dans la cellule où ils sont liés à la CRABP (cytoplasmic retinoic acid binding protein); ils sont transférés jusqu'au noyau où ils sont reçus par des récepteurs nucléaires. La CRABP est-elle utile dans ce transfert ou joue-t-elle le rôle d'appât qui perturbe ce transfert ? Cela est en cours d'étude.

Les acides rétinoïques ont une action principale sur deux phénomènes : le développement embryonnaire de tissus variés et la transformation maligne. Ces substances peuvent stimuler ou inhiber de très nombreuses enzymes. Ce sont donc de grands modulateurs de l'expression génique.

\section{Les récepteurs nucléaires des acides rétinoïques}

Les récepteurs nucléaires des acides rétinoïques ont une structure similaire à celle des récepteurs des hormones stéroïdes, thyroïdiennes et de la vitamine D3. Il s'agit de récepteurs nucléaires qui se fixent à des séquences spécifiques d'ADN. Quatre gènes différents de ces récepteurs ont été à ce jour clonés : $\alpha$ [5], $\beta$ [6], $\gamma$ [7] et $R A R X$ [8]. Ils ont des profils de transcription distincts suivant les tissus ; en particulier, l'ARN messager du récepteur $\gamma$ est spécifiquement présent au niveau des cellules de la peau, celui du récepteur $\alpha$ est majoritaire dans les cellules myéloïdes et celui du récepteur $\beta$ dans de nombreux tissus (foie, cerveau, rein...).

L'expression du gène du récepteur $\beta$ est contrôlée par la concentration de l'acide rétinoïque lui-même qui en augmente la transcription dans des hépatocytes [9]. Il existe, en effet, un élément de réponse à l'acide rétinoïque à proximité du promoteur du gène, élément sur lequel se fixe le complexe acide rétinoïque/récepteur [10].

Le gène $\mathrm{du}$ récepteur $\alpha$ est localisé sur le chromosome $17 q 21$, donc à proximité de la translocation $15 ; 17$, spécifique de la leucémie aiguë promyélocytaire. Le gène du récepteur $\beta$ est, en revanche, situé dans la région $3 \mathrm{p} 24$ et le gène $\gamma$ sur le chromosome 12 .

\section{Effets \\ des acides rétinoïques sur des cellules provenant de malades leucémiques}

Les effets sur des lignées cellulaires in vitro. Les acides rétinoïques induisent la différenciation irréversible de certaines cellules malignes. Les modèles les plus étudiés de cet effet sont ceux des lignées cellulaires de tumeur du sein, du tératocarcinome F9 murin et des lignées de cellules leucémiques myéloïdes humaines.

Sous l'effet d'agents différenciants, les lignées de cellules leucémiques peuvent être engagées in vitro vers une différenciation terminale et fonctionnelle au terme de laquelle la cellule mature meurt. Il y a donc induction du programme de mort cellulaire. Même si, parfois, les cellules n'ont pas acquis toutes les caractéristiques d'une cellule normale, elles ont au moins perdu celles de malignité. Les acides rétinoïques, et principalement l'acide touttrans rétinoïque, sont des inducteurs particulièrement puissants de la différenciation des lignées de cellules malignes myéloïdes HL60 [11] et monocytaires de type U937 ou THP1.

Les effets in vitro sur des cellules de malades atteints de leucémie. L'acide tout-trans rétinoïque agit sur les cellules fraîches provenant de malades atteints de leucémie aiguë promyélocytaire, provoquant leur différenciation en cellules fonctionnelles. Parmi 40 malades étudiés, atteints de différentes formes de leucémie aiguë myéloblastique, seules les 11 cas de leucémie aiguë promyélocytaire répondaient à l'induction de la différenciation par l'acide rétinoïque [12]. Les cellules ainsi traitées acquièrent un aspect cytologiquement " normal " de cellules matures, une fonction phagocytaire, et renforcent l'expression du marqueur CD15.

Il existe une importante différence d'effet suivant les dérivés d'acide réti- 


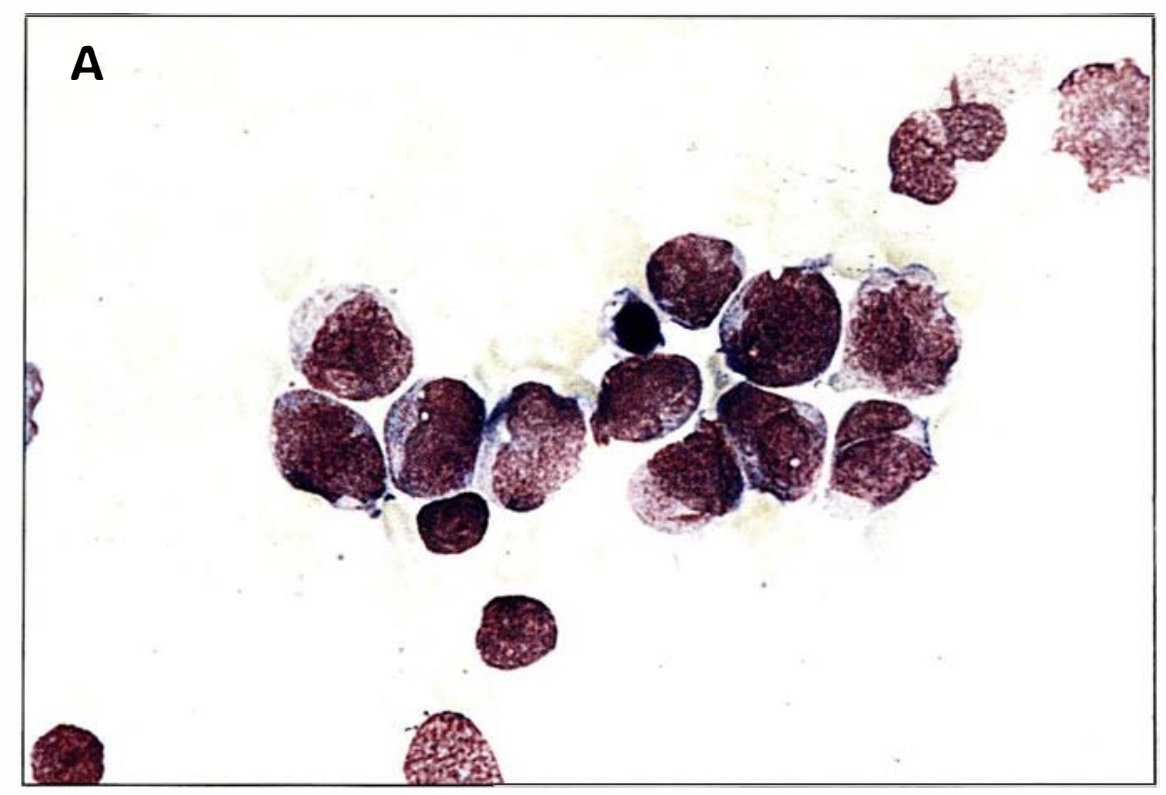

\section{RÉFÉRENCES}

1. Sachs L. Control of normal cell differentiation and the phenotypic reversion of malignancy in myeloid leukemia. Nature 1978 ; $274: 535-9$

2. Housset M, Daniel MT, Degos L. Small doses of Ara-C in the treatment of acute myeloid leukemia : differentiation of myeloid leukemia cells ? Br J Hematol 1982 ; 51 : $125-9$.

3. Tilly $\mathrm{H}$, Castaigne $\mathrm{S}$, Bordessoule $\mathrm{D}$, et al. Low-dose cytarabine versus intensive chemotherapy in the treatment of acute nonlymphocytic leukemia in the elderly. $J$ Clin Oncol 1990 ; 8 : 272-9.

4. Genot E, Billard C, Signaux F, et al. Proliferative response of hairy cells to B cell growth factor (BCGF) : in vivo inhibition by interferon $\alpha$ and in vivo effects of interferon $\alpha, \beta$, and $\gamma$. Leukemia $1987 ; 1$ : 590-6.

5. Giguere V, Ong ES, Segui $P$, Evans RM. Identification of a receptor for the morphogen retinoic acid. Nature 1987 ; $33: 624-9$.

6. Petkovich M, Brand NJ, Kurst A, Chambron P. A human retinoic acid receptor which belongs to the family of nuclear receptors. Nature 1987 ; 330 : 444-50.

7. De Thé $H$, Marchio A, Tiollais $P$, Dejean A. A novel steroid thyroid receptorrelated gene inappropriately expressed in human hepatocellular carcinoma. Nature

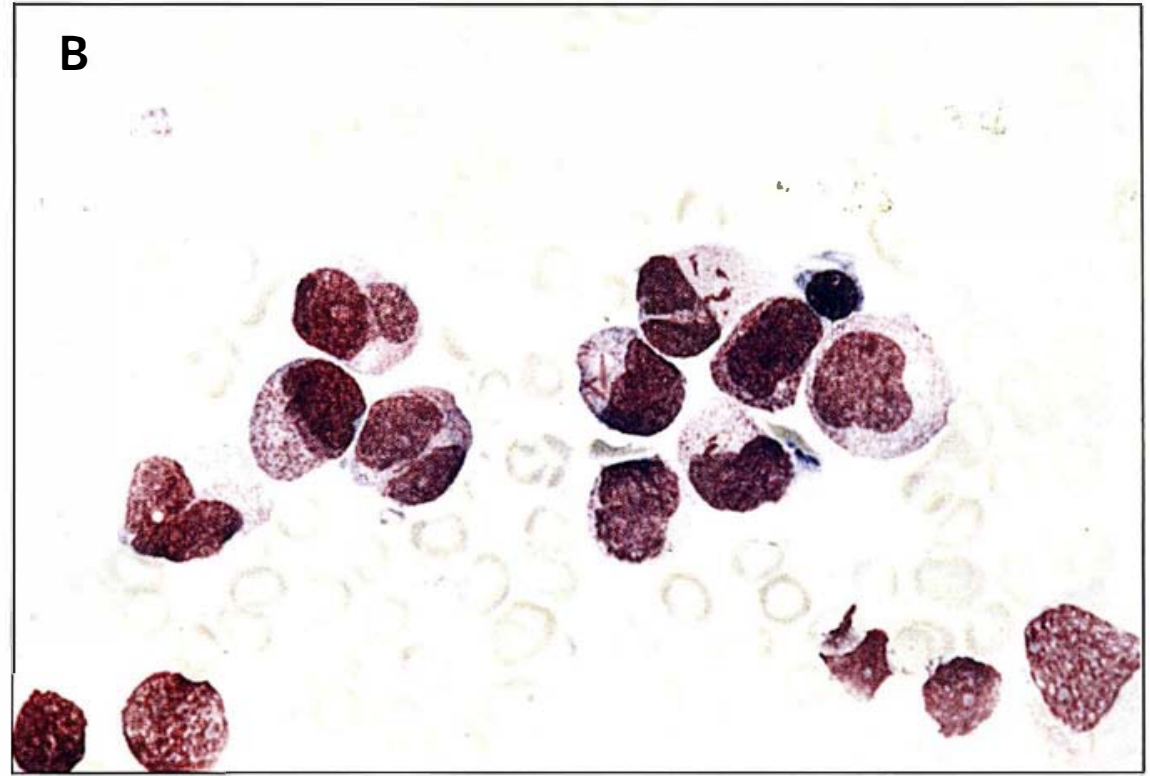

noïque. La différenciation maximale est obtenue avec une concentration de $10^{-7} \mathrm{~mol} / \mathrm{l}$ pour l'acide tout-trans rétinoïque et de $10^{-6} \mathrm{~mol} / \mathrm{l}$ pour l'acide 13-cis [12]. L'étrétinate (ou ester-éthyl d'acide rétinoïque) est sans effet.

Traitement des malades atteints de leucémie aiguë promyélocytaire

Deux dérivés de l'acide rétinoïque sont actuellement disponibles en thérapeutique humaine: l'étrétinate (Tigason $^{\circledR}$ ) et l'acide 13-cis rétinoïque (Roaccutane $^{\circledR}$ ).

L'étrétinate paraît inefficace in vivo pour traiter ces malades alors que seule une faible proportion de malades a été mise en rémission complète pour un traitement par l'acide 13-cis rétinoïque $\left(100 \mathrm{mg} / \mathrm{m}^{2} / \mathrm{j}\right)$.

L'acide tout-trans rétinoïque a été d'abord fabriqué en Chine et un essai thérapeutique mené par l'uni- 


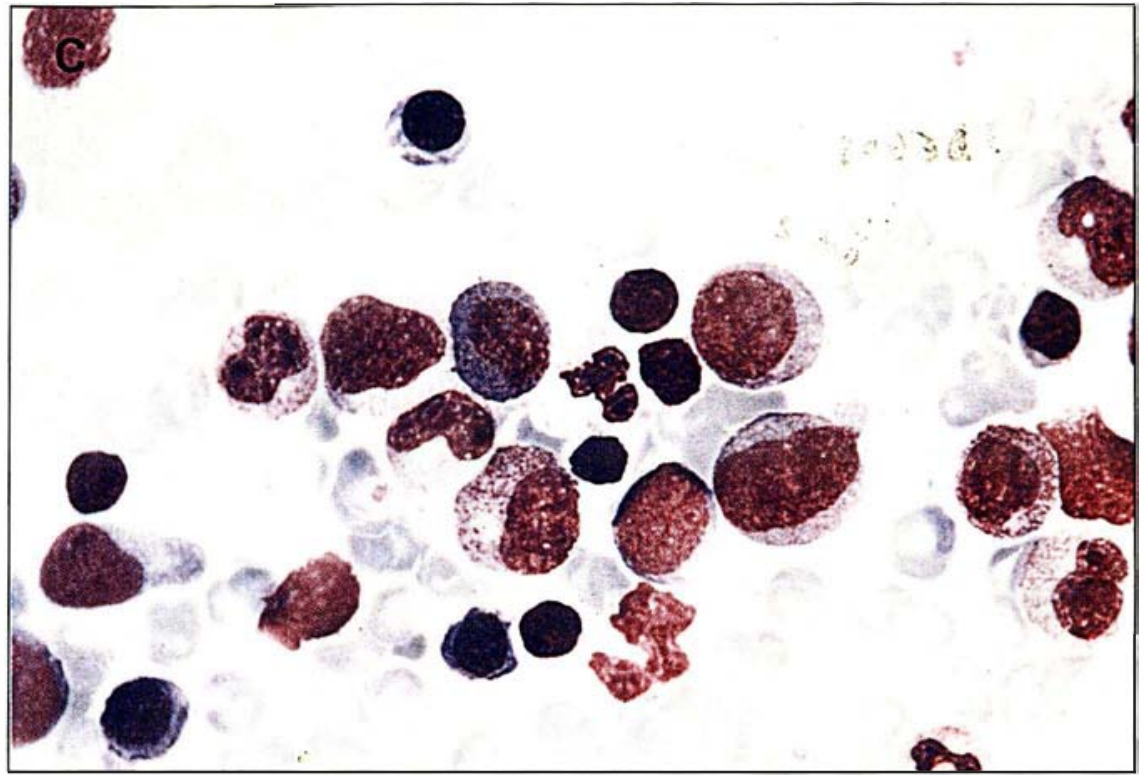

Figure 1. Évolution de l'état médullaire durant le traitement de la leucémie aiguë promyélocytaire par l'acide tout-trans rétinoïque. Photo $A$ : avant traitement. Photo $B$ : après un mois. Photo $C$ : après trois mois de traitement. Les cellules deviennent matures de manière progressive (clichés M.T. Daniel).

versité de Shanghaï $\mathrm{n}^{\circ} 2$ sur 24 malades atteints de leucémie aiguë promyélocytaire a montré sa grande efficacité en induisant 23 rémissions complètes [13].

L'acide tout-trans rétinoïque produit par les Chinois, puis par Roche France (depuis juin 1989) a été aussi proposé aux malades atteints de ce type de leucémie aiguë en France [14]. Les malades avaient une contreindication à la chimiothérapie intensive ou étaient en rechute de leur maladie. La fréquence de rémission complète a été de $71 \%$ dans une première étude de 34 malades. Les rémissions partielles survenaient lors du traitement pour deuxième rechute et la seule résistance a été notée chez un malade en troisième rechute. Des décès précoces ont été rapportés chez des personnes qui ont eu une hyperleucocytose supérieure à 30000 globules blancs $/ \mathrm{mm}^{3}$. Une étude plus particulière du traitement des malades en première rechute [15] a révélé que $95 \%$ des malades obtiennent une rémission complète. Une étude pilote pour un essai européen a aussi montré l'efficacité de ce traitement (95\% de rémission complète) chez des malades, traités en première intention, mais en prenant des pré- cellules leucémiques est progressive. Les cellules blastiques subissent une maturation qui va jusqu'à une différenciation terminale. Parfois, on trouve même des corps d'Auer dans des cellules mûres ressemblant aux polynucléaires. Tandis que les cellules se différencient, la moelle se normalise aussi bien du point de vue cytologique que cytogénétique, sans phase d'hypocellularité. Les cultures à court terme de cellules leucémiques (5 jours), en présence d'acide rétinoïque, permettent de prédire l'effet in vivo car il existe une bonne corrélation entre les différenciations in vitro et in vivo [12].

Les transfusions ne sont généralement pas nécessaires (dans $75 \%$ des cas). L'héparine, en dehors des crises de coagulation intravasculaire disséminée, n'est prescrite que dans un petit nombre de cas $(10 \%)$ et les conditions stériles de traitement ne sont pas requises. Seulement $20 \%$ des malades ont reçu des antibiotiques. En première rechute, la durée d'hospitalisation a été inférieure à une semaine dans $75 \%$ des cas.

La toxicité du traitement semble faible, marquée seulement par une sécheresse de la peau et des muqueuses chez tous les malades, des douleurs osseuses traitées par des antalgiques dans $35 \%$ des cas et une augmentation des transaminases hépatiques $(20 \%)$ et des triglycérides (55\%), anomalies toujours réversibles.

Cependant un événement rare en première rechute (18\% des cas), fréquent en traitement de première intention (35\% des cas), l'hyperleucocytose, apparaît être une complication grave du traitement par l'acide tout-trans rétinoïque. Cette hyperleucocytose survient entre le $5^{\mathrm{e}}$ et le $20^{\mathrm{e}}$ jour et doit être considérée comme un événement dangereux puisqu'elle s'associe à la réapparition de la coagulation intravasculaire et que trois quarts des malades qui ont eu une forte hyperleucocytose (au-dessus de 30000 globules blancs $/ \mathrm{mm}^{3}$ ), sont décédés. Pendant cette phase, les globules blancs sont en voie de maturation. Il n'y a donc pas d'échec thérapeutique et, dans les cas où les malades ont survécu, les globules blancs ont progressivement diminué lors de la poursuite du traitement par 
l'acide rétinoïque, la rémission complète étant obtenue dans un délai normal. C'est pourquoi il est actuellement proposé d'ajouter une chimiothérapie dès que les globules blancs dépassent $5000 / \mathrm{mm}^{3}$ en cinq jours, $10000 / \mathrm{mm}^{3}$ en dix jours ou $15000 / \mathrm{mm}^{3}$ en 15 jours.

La raison de cette hyperleucocytose durant le traitement n'est pas claire. Il s'agit généralement de malades traités en première intention, qui ont une infiltration importante de la moelle et qui ont eu une forte coagulation intravasculaire dissiminée au début du traitement.

Les rechutes surviennent malheureusement dans des délais relativement courts (médiane de 8 mois). C'est pourquoi nous proposons actuellement des traitements associant une chimiothérapie, une fois la rémission complète obtenue par l'acide touttrans rétinoïque. Lors des rechutes après ce traitement, les cellules malignes sont résistantes à l'acide rétinoïque. Un travail est en cours pour comprendre la résistance acquise à l'acide rétinoïque.

L'activité spécifique de l'acide touttrans rétinoïque sur les cellules de leucémie aiguë promyélocytaire pose différentes questions : les effets biologiques sont-ils liés à la localisation du point de cassure sur le chromosome 17 au niveau du gène du récepteur $\alpha$ de l'acide rétinoïque [16]? On sait que le remaniement $t(15 ; 17)$ entraîne la formation d'un gène hybride [17] comprenant le motif de liaison à l'ADN et le domaine de liaison de l'acide rétinoïque du récepteur, liés à d'autres séquences provenant du gène myl du chromosome 15 . On ne sait pas quelle est la fonction de la protéine hybride correspondante. Joue-t-elle un rôle dans la leucémogenèse ? Dans le blocage de différenciation au stade promyélocyte? Quel est le mécanisme d'action de l'acide rétinoïque lorsqu'il permet à nouveau une différenciation?

En conclusion, la leucémie aiguë promyélocytaire, une forme sévère de leucémie aiguë, est traitée avec efficacité par l'acide tout-trans rétinoïque. Une fréquence élevée de rémission complète est obtenue même dans les cas de rechute. La base moléculaire précise de cet effet reste encore inconnue, même si le remaniement du gène du récepteur $\alpha$ de l'acide rétinoïque est probablement impliqué; l'acide tout-trans rétinoïque apparaît en tout cas être la cause de la maturation des cellules malignes. Il s'agit donc du premier cas de succès dans le traitement du cancer par induction de la différenciation des cellules malignes

\section{Summary}

All-trans retinoic acid in the treatment of acute promyelocytic leukemias

Acute promyelocytic leukemia is a rare and severe leukemia, associated with a disseminated intravascular coagulation (DIC) and a specific $\mathrm{t}(15 ; 17)$ translocation. All-trans retinoic acid, a vitamin $\mathrm{A}$ derivative, is able to induce complete remission via a differentiation effect in almost all cases of first attack (Chinese and French results) or in first relapse (French data). Proliferation is not stopped during the first weeks of treatment and hyperleucocytosis is a major adverse effect. This treatment is given orally, generally without any infusion or transfusion. Patients are treated as out-patients. DIC rapidly disappears, and normal cells progressively replace the abnormal maturing cells. All-trans retinoic acid induces differentiation of malignant cells. These cells are then engaged in maturation process leading to an apparent normal phenotype. This is the first model of differentiation therapy in malignancy with a high complete remission rate.
TIRÉS A PART
L. Degos.

TIRÉS A PART
L. Degos.

$m / s n^{\circ} 5$, vol. 7, mai 91 